\title{
Erratum to: Hyperbolic metamaterials-based plasmonic biosensor for fluid biopsy with single molecule sensitivity
}

\author{
EPJ Appl. Metamat. 2017, 4, 1, DOI: 10.1051/epjam/2016015
}

Kandammathe Valiyaveedu Sreekanth ${ }^{1}$, Mohamed ElKabbash ${ }^{1}$, Yunus Alapan ${ }^{2}$, Efe Ilker ${ }^{1}$, Michael Hinczewski ${ }^{1}$, Umut A. Gurkan ${ }^{2,3,4,5}$, and Giuseppe Strangi ${ }^{1,6,7,8, *}$

1 Department of Physics, Case Western Reserve University, 10600 Euclid Avenue, Cleveland, OH 44106, USA

2 Case Biomanufacturing and Microfabrication Laboratory, Mechanical and Aerospace Engineering Department, Case Western Reserve University, Cleveland, OH 44106, USA

3 Biomedical Engineering Department, Case Western Reserve University, Cleveland, OH 44106, USA

4 Department of Orthopedics, Case Western Reserve University, Cleveland, OH 44106, USA

5 Advanced Platform Technology Center, Louis Stokes Cleveland Veterans Affairs Medical Center, Cleveland, OH 44106, USA

6 CNR-NANOTEC Istituto di Nanotecnologia and Department of Physics, University of Calabria, 87036 Rende, Italy

7 Case Comprehensive Cancer Center, Case Western Reserve University, Cleveland, OH 44106, USA

8 IIT, Italian Institute of Technology, Via Morego 30, 16163 Genova, Italy

A mistake happened during production: the name of the fourth author Efe Ilker was repeated (Efe Ilker Ilker).

The correct name must be Efe Ilker. The publisher apologises for this error.

Cite this article as: Sreekanth K.V., ElKabbash M, Alapan Y, Ilker E, Hinczewski M, Gurkan UA \& Strangi G: Erratum to: Hyperbolic metamaterials-based plasmonic biosensor for fluid biopsy with single molecule sensitivity. EPJ Appl. Metamat. $2017,4,4$.

*e-mail: giuseppe.strangi@case.edu

This is an Open Access article distributed under the terms of the Creative Commons Attribution License (http://creativecommons.org/licenses/by/4.0), which permits unrestricted use, distribution, and reproduction in any medium, provided the original work is properly cited. 